\title{
The Influence of Political Propaganda on Space Activities: a Case Study
}

\section{of Dongfanghong-I}

\author{
Liu $\operatorname{Lin}^{1, a^{*}}$, Zeng Guang ${ }^{2, b}$ \\ ${ }^{1}$ College of Humanities and Social Sciences, National University of Defense Technology, \\ 410073, Changsha, P.R. China \\ ${ }^{2}$ College of Humanities and Social Sciences, National University of Defense Technology, \\ 410073, Changsha, P.R. China \\ a125445236@qq.com, bouangzeng92@163.com
}

Keywords: Dongfanghong-I, observable spheroid, orbit, electronic music generator, overload switch

\begin{abstract}
The development of science and technology, especially the major events, are not only affected by purely technical factors, but also bound up with many irrational social factors. Space activities, of significant importance in science and technology as well as in the political field, are the combination of inner and outer factors, which reflect comprehensive strength of the nation. With the purpose of analyzing the influence of social construction on technological development, the author focuses on the case of Dongfanghong-I, making explanation and analysis of the influence of politics propaganda on the research and development of the satellite in the historical context. The paper concentrates on three aspects: the proposal of propaganda function of Dongfanghong-I, the transition of the tasks of the satellite and its particular propaganda functions; specifically in terms of how political propaganda affects satellite technology such as observable spheroid, orbit, electronic music generator and overload switch.
\end{abstract}

Space activities, being the aggregate of a wide range of disciplines and technologies, are reflections of comprehensive national strength, which reflects the country's economic, scientific and technological, as well as military strength. Not only do the significant strides in space industry represent a breakthrough at the technical level, but also political significance. Especially in particular historical periods, space activities carry an important mission of political propaganda and their underlying motives are principally political rather than pure technical ones. The first man-made satellite of China, "Dongfanghong-I", came into being in the Cultural Revolution. So, all aspects of the whole project, from design to launch, is marked by that period, and to a great extent, political propaganda influences and determines the satellite program.

Internationally, The 1950s witnessed the struggle for hegemony between America and the Soviet Union, and there was a fierce confrontation between capitalism and socialism, especially for space supremacy. On October $4^{\text {th }}, 1957$, the Soviet Union successfully launched the world's first artificial satellite "Sputnik 1". The United States was spurred to regain a technological lead and lunched the artificial satellite "Explorer 1" on January 31st, 1958. All the endeavors above marked that the focus of aeronautic technology shifted to the space. Domestically, Taiwan became "swollen with arrogance", fueled by supports of the United States. At the same time, China's legitimate seat in the United Nations was strongly opposed by the United States government. China's sovereignty was seriously threatened. 
Under such circumstances, China's leaders headed by Mao Zedong had a profound insight of the importance of developing space industry, which was not only seemed as a competition of science and technology, but also a political battle. From then on, China has started her own space program.

During the 2nd Plenary Session of the 8th CPC Central Committee in May, 1958, Mao Zedong twice empathized the issue of satellite. During the meeting for the construction project of the first artificial satellite in 1965, also called "651meeting", delegates highlighted that political impacts should come in the first place. In 1967, the overall goal of the satellite project was set, which could be summed up in four words: feasible, controllable, visible and audible. [1]

During the development of the satellite, all technical problems were closely related to the major mission of political propaganda. Especially the three significant objectives, which corresponded to propaganda, "controllable", "visible" and "audible" were respectively reflected in the design of satellite's "observable spheroid", "orbit planning", as well as "electronic music generator" and "overload switch".

\section{The invention and improvement of observable spheroid}

"Visible" means that during "Dongfanghong-I" traveling in the orbit, it should be visible for naked eyes around the world, which is beneficial to expanding range of influence and reinforcing the propaganda effect. However, actually, it is really difficult to see the satellite with the naked eye, since human eyes can only see the objects of $2^{\text {nd }}$ to 3 rd magnitude. But the visibility of "Dongfanghong-I" only ranges from 5th to 8th magnitude when orbiting, which is far beyond human being's ability to observe. So, there are two technical difficulties towards achieving the goal of being "visible": the first one is to attach observable spheroid to the satellite to increase the brightness of it; and the second is to change the original orbit and increase the degree of inclination so as to expand the visible range.

The diameter of the Satellite "Dongfanghong-I" itself is only 1 meter, barely visible to the naked eyes; in order to enhance the satellite's reflective effect, the shape of satellite was designed to be a polyhedron of 72 sides. Even so, it was still difficult to follow the satellite with the naked eyes when the weather was not good since the satellite was too small. So experts decided to attach the "observable spheroid" to the third-stage of the launch vehicle. After the satellite was launched into the orbit, the "observable spheroid" would be deployed and turned into a sphere with the diameter of 3-4 meters, whose surface is plated with aluminum. Through the reflection of sunlight, the visibility of the satellite (actually the third-stage of the launcher) would rise up to $2^{\text {nd }}-3^{\text {rd }}$ magnitude. [2] In fact, what the watchers see was not the satellite itself, but its "observable spheroid" installed to the third-stage rocket launcher, which has the same orbit as the satellite. But scientists encountered another technical difficulty in developing the "observable spheroid"-when the "observable spheroid" was added, the total weight of the satellite would exceed the carrying capacity of the launcher. Since the rocket launcher had already been put into production at that time, the only way was to limit the weight of the "observable spheroid", which should be less than $17.5 \mathrm{~kg}$.

Through inspection and analysis over and over again, experts found the key to the problem: three major components made up most of the weight of "observable spheroid": guide rod, metallic ring added to the bottom and the large spring. At the beginning, the guide rod was made of stainless steel tubes. After intense work of scientific exploration and analysis, it was substituted by aluminum alloy; also the spring was removed and the projectile was done by virtue of compressed gas. After a series of improvements and modifications, the "weight problem" of the "observable spheroid" was 
solved. [3] Finally the task to develop the "observable spheroid" was accomplished as scheduled, at the beginning of 1969. [4]

\section{The measurement and selection of orbit}

There were mainly two issues with the orbit - the measurement and selection. Measurement of the orbit was very important, because timely tracking of the satellite's orbit and accurately positioning the satellite were directly related to the realization of the target of "controllable". We must track and locate the satellite as soon as possible and calculate the accurate time when it flew over China. Once we did not detect the satellite during the period, and the trajectory was found by other countries earlier, it would be a serious political accident. However, compared with the Soviet Union, the territory of China only spans the width of 60 latitudes; meanwhile, China could not establish ground stations all over the world as the United States did. So the orbit measurement could only be realized based on China's own territory and by short arc orbit determination. Experts broke the traditional geometric mode of thinking, and derived a set of independent equations for orbit measurement. Furthermore, they carried out the short-arc segment tracking simulation and tracking research, whose results showed the feasibility Doppler measurement of the orbit based on independent tracking by multi-station. [5]

In the 1960s, China had only one satellite launch center, Jiuquan. Limited by the launch site and the falling regions of the first and second stage launcher, and in order to take advantage of the power generated by the earth's rotation to increase the payload, it was initially planned to launch the satellite 80 degrees north east. If so, however, the angle between the satellite orbit and the equator was only 42 degrees, which meant that only people in mid or low latitude regions could see the satellite orbiting, while many countries in North America and Europe could not at all. It would heavily weaken the political propaganda effect of launching satellite. In order to increase the area of observation and better complete the target of "visible", it was necessary to reselect the orbit. During "The Meeting to Select the Orbit" held in April, 1966, experts compared three proposals - the inclination angle of 42 degrees, 60 degrees to 70 degrees and 90 degrees, and finally decided to change the orbital plane inclination to 70 degrees and change the launching direction to 70 degrees east by south, which was the direction from Jiuquan to Hainan Island. [6]

\section{Electronic music generator}

"Audible" meant that the satellite should transmit continuous radio signals of the Chinese music Dongfanghong after it was launched, which was in its essence, a political task that it implies to make the world hear the voice of the socialist countries, and the voice of China demonstrating the growing comprehensive national strength and the superiority of the socialist system. The music and dance epic Dongfanghong created in 1964 had created a great sensation. As a classic red song, it revealed worship and gratitude of Chinese people to Chairman Mao and their belief and passion in socialism.

There were different plans to generate the musical sound of Dongfanghong, and after rounds of comparison and discussion, it was finally decided to use electronic music since it is reliable, durable, of low power consumption and the sound is loud and clear. [7] Therefore, the development of electronic music generator became the priority for all. Initially, experts tried to employ a transmitter to send out signal of "Dongfanghong", but the weight of such a high-power transmitter should be more than 1 tons to ensure that it was audible from the ground, which heavily exceeded the payload of the launcher. In the end, the experts decided to cooperate with the central broadcasting station. High-power stations on the ground received the signals and then distributed it with the help of broadcast system, so that people around the world could hear the voice of China. [8] 
At the same time, the accuracy of time and tone of the music could never be underestimated. Once in a pilot broadcast, there was a modulation of the music, which worried the scientists a lot that once it happened in the real launching process, it would be a great shame, which would cause severe political influences, not mentioned to realize due propaganda effect. It turned out that electron beam resulted from the fierce irradiation would give rise to electromagnetic interference, and then distorted the musical syllables. Besides, the tone would be altered if the temperature was too high or too low. Having experienced repeated trials and errors, Chief Superintendent, Mr. Liu Chengxi, shouldering great responsibility and stress, finally found the solution-embedded sealing, to keep the temperature stable. [9] Furthermore, experts also turned to music professionals, and agreed on the adoption of electronic music generated by circuit stimulation.

In the second half of 1968, the music Dongfanghong was played accurately in test broadcasts, which marked the success of electronic music generator.

\section{The installation and cancellation of the overload switch}

It was a prerequisite for the successful transmission of musical signals of Dongfanghong that the satellite enters into the orbit, which depended on the fact that the third-stage rocket launcher reached the first cosmic velocity (V1). Even if the music was played without a hitch, but at a wrong time or place, it would mess the project up under the watchful eyes of people around the world; and this will end up not an opportunity to demonstrate the achievements and success of China, but an ironic, farce, which would be a serious political problem that might influence each expert. In order to prevent such a situation, Mr. Qian Xuesen came up with the proposal to install an addition "overload switch" on the final stage.

"Overload switch" was an insurance device which can shut down the system and cut off the circuit when the music was not supposed to play. That was to say, if the third-stage reached the V1, the "overload switch" and the circuit generating "Dongfanghong" music was connected, then everything went as planned and the music would be played sound and clear; but if the launcher failed to reach the V1, or something else went wrong, the "overload switch" would be turned off, and the circuit for "Dongfanghong" music would not be connected; in this case, there would be no music, and the satellite project would be a silent failure. [10]

However, any technology comes with risk and benefit. Once the "overload switch" itself went wrong, the music would not be played in due time or even not played at all. This was another thorny problem that aroused controversy. Some experts claimed to retain the "overload switch", while others advocated cancellation. Such a tiny switch perplexed the experts and there had been no consensus for a long time. On April 14th, 1970, in the work report concerning satellite construction submitted to Premier Zhou Enlai, Qian Xuesen reported the discussion of the "overload switch". Having been informed of the reliability of the satellite and rocket launcher, Premier Zhou instructed to remove the "overload switch". [11] Actually, the discussion about the "overload switch" was not only a purely scientific issue, but also a technical problem of political significance, involving different phases of satellite construction and various departments of the central authority.

On April 24, 1970, the satellite of "Dongfanghong-I" was successfully launched. When it entered into the orbit, ground stations tracked and positioned it in time and accurately broadcasted the time when it passed over the capitals of different countries. The music "Dongfanghong" sounded loud and clear in space that people around the world could hear it on broadcast. With this, the "Dongfanghong-I" successfully completed the stated objective of "feasible", "controllable", "visible" and "audible". The successful launch of "Dongfanghong-I" had a great political propaganda significance. It did not only inspire Chinese people with enthusiasm, but also showed 
the national strength of China, and contributed to the enhancement of China's international status.

"Dongfanghong-I" was constructed in a particular historical period and deeply marked by that time. Such a case of "Dongfanghong-I" project fully demonstrated the significant influence of political propaganda on space activities. In fact, factors affecting the development of technology are complex, the author only analyzed the interaction between political propaganda and science and technology, and there are many other social factors that deserve further study.

\section{References}

[1] Yang Zhaode, The cultural significance of launching satellite-- the 35 year anniversary of the first man-made satellite in China, The National Defense Science and Technology Industry Press, Beijing, 2005(4):26-27.

[2] Please remember their history - Chinese scientists and "Hydrogen bombs", Science Times, Jinan University Press, Guangzhou, 1999.

[3] Contemporary China's space industry Editorial Committee, contemporary China's aerospace industry, China Social Sciences Press, Beijing, 1986.

[4] Lin Xianyou, A new star in space, Space Industry Management, Beijing, 2005 (05): 13-14.

[5] Yang Zhaode, "Dongfanghong- I " -the story of China's first satellite, Military Culture, 2014 (09): 86-89.

[6] Li Yili, the optimization of China's first satellite launch vehicle overall program, Space Environment and Engineering, 2015 (04): 130-134.

[7] Shanghai Astronomical Observatory.CAS, artificial earth satellite, Shanghai Publishing Revolution Group, Shanghai, 1970.

[8] Hang Tianren, China's first man-made earth satellite story, Knowledge is Power, 2015 (04): 50-53.

[9] Sun Jiadong, Hu Xueqin, " Dongfanghong- I"—which can not be stopped by the "Cultural Revolution" , China Economic Weekly, 2009 (09): 42-43.

[10] Li Mingsheng, The rise of the first star - " Dongfanghong- I", Youth Literature, 1995 (07): $5-20$.

[11] Li Mingsheng, Get out of the earth Chinese village - the first artificial earth satellite heaven, Jinqiu Keyuan, 1996 (07): 16-18. 